\title{
SINGULAR INTEGRALS RELATED TO HOMOGENEOUS MAPPINGS IN TRIEBEL-LIZORKIN SPACES
}

\author{
FENG LIU AND HUOXIONG WU
}

\begin{abstract}
In this note we establish the boundedness for the singular integral operators related to homogeneous mappings with rough kernels in Triebel-Lizorkin spaces. Some previous results are improved and extended substantially. A main ingredient in the proofs is to establish a criterion of boundedness for the convolution type operator in the above function spaces, which presents a systematic treatment for the related singular integral operators.
\end{abstract}

Mathematics subject classification (2010): 42B20, 42B25, 42B15, 42B30.

Keywords and phrases: Singular integrals, homogeneous mappings, rough kernels, Triebel-Lizorkin spaces.

\section{REFERENCES}

[1] H. AL-QASSEM, On the boundedness of maxiaml operators and singular operators with kernels in $L\left(\log ^{+} L\right)^{\alpha}\left(S^{n-1}\right)$, J. Inequal. Appl. 2006, 1 (2006), 1-16.

[2] H. Al-QAssem, A. Al-S Alman And Y PAn, Singular integrals associated to homogeneous mappings with rough kernels, Hokkaido Math. J. 33, 3 (2004), 551-569.

[3] H. Al-QAssem, L. Cheng And Y. PAN, Boundedness of rough integral operators on TriebelLizorkin spaces, Publ. Math. 56, 2012 (2012), 261-277.

[4] A. Al-Salman And Y. PAn, Singular integrals with rough kernels in $L \log L\left(S^{n-1}\right)$, J. London Math. Soc. 66, 2 (2002), 153-174.

[5] Y. CHen AND Y. Ding, Rough singular integrals on Triebel-Lizorkin space and Besov space, J. Math. Anal. Appl. 347, 2 (2008), 493-501.

[6] Y. Chen, Y. Ding AND H. LiU, Rough singular integrals supported on submanifolds, J. Math. Anal. Appl. 368, 2 (2010), 677-691.

[7] L. Cheng, Singular ingegrals related to homogeneous mappings, Michigan Math. J. 47, 1 (2000), 407-416.

[8] A. Calderón And A. Zygmund, On singular integrals, Amer. J. Math. Soc. 78, 2 (1956), 289-309.

[9] J. Chen And C. Zhang, Boundedness of rough singular integral on the Triebel-Lizorkin spaces, J. Math. Anal. Appl. 337, 2 (2008), 1048-1052.

[10] R. Coifman And G. Weiss, Extension of Hardy spaces and their use in analysis, Bull. Amer. Math. Soc. 83, 1 (1977), 569-645.

[11] L. Colzani (Eds), Hardy spaces on spheres, PhD thesis, Washington University, St. Louis, 1982.

[12] Y. Ding, Q. XUE AND Y. YABUTA, On singular interal operators with rough kernel along surfaces, Integr. Equa. Oper. Theory 68, 1 (2010), 151-161.

[13] Y. Ding AND Y. YABUTA, Triebel-Lizorkin space boundedness of rough singular integrals associated to surfaces of revolution, Sci. China Math. 59, 9 (2016), 1721-1736.

[14] J. DuoAndikoetXeA And J. Rubio De Francia, Maximal and singular integral operators via Fourier transform estimates, Invent. Math. 84, 3 (1986), 541-561.

[15] D. FAN, K. GUO AND Y. PAN, $L^{p}$ estimates for singular integrals associated to homogemeous surfaces, J. reine angew Math. 542, 1 (2002), 1-22.

[16] D. FAN AND Y. PAN, Singular integral operators with rough kernels supported by subvarieties, Amer. J. Math. 119, 4 (1997), 799-839. 
[17] D. FAN AND H. WU, Non-isotropic singular integrals and maximal operators along surfaces of revolution, Math. Inequal. Appl. 16, 2 (2013), 461-476.

[18] R. Fefferman, A note on singular integrals, Proc. Amer. Math. Soc. 74, 2 (1979), 266-270.

[19] M. FRAZIER AND B. JAWERTH, A discrete transform an decompositions of distribution spaces, J. Funct. Anal. 93, 1 (1990), 34-170.

[20] M. FraZIER, B. JAWERTH AND G. Weiss, Littlewood-Paley thorey and the study of Function Spaces, CBMS Reg. Conf. Ser. 79, Amer. Math. Soc., Providence, RI, 1991.

[21] L. Grafakos, Classical and Modern Fourier Analysis, Prentice Hall Upper Saddle River, NJ, 2003.

[22] L. HÖRMANDER, The analysis of linear partial differential operators I, Springer-Verlag, Berlin, 1983.

[23] F. LiU, Integral operators of Marcinkiewicz type on Triebel-Lizorkin spaces, Math. Nachr. 291, 1 (2017), 75-96.

[24] F. LiU AND H. WU, Rough singular integrals supported by submanifolds in Triebel-Lizorkin spaces and Besov spaces, Taiwanese J. Math. 18, 1 (2014), 127-146.

[25] F. LIU AND H. WU, Boundedness of certain singular integrals along surfaces on Triebel-Lizorkin spaces, Forum Math. 27, 6 (2015), 3439-3460.

[26] F. LiU, S. MAO AND H. WU, On rough singular integrals related to homogeneous mappings, Collect. Math. 67, 1 (2016), 113-132.

[27] F. LiU, H. WU AND D. ZHANG, A note on rough singular integrals in Triebel-Lizorkin spaces and Besov spaces, J. Inequal. Appl. 2013, 492 (2013), 1-13.

[28] W. LI, Z. Si AND K. YABUTA, Boundedness of singular integrals associated to surfaces of revolution on Triebel-Lizorkin spaces, Forum Math. 28, 1 (2016), 57-75.

[29] Y. SAWANO AND K. YABUTA, Fraction type Marcinkiewicz integral operators associated to surfaces, J. Inequal. Appl. 2014, 232 (2014), 1-29.

[30] H. TRIEBEL, Theory of Function Spaces, Monogr. Math. vol. 78, Birkhäser Verlag, Basel, 1983.

[31] Q. XUE, K. YABUtA AND J. YAN, Fractional type Marcinkiewicz integral operators on function spaces, Forum Math. 27, 5 (2015), 3079-3109.

[32] K. YABUTA, Triebel-Lizorkin space boundedness of rough singular integrals associated to surfaces, J. Inequal. Appl. 2015, 1 (2015), 1-26.

[33] K. YaBUtA, Triebel-Lizorkin space boundedness of Marcinkiewicz integrals associated to surfaces, Appl. Math. J. Chinese Univ. Ser. B 30, 4 (2015), 418-446. 